\title{
Kommunale Praxis verändern
}

\author{
Mario Rund und Ronald Lutz
}

\begin{abstract}
Die Stadtplanung in vielen großen Kommunen geht vom Leitbild des »Unternehmens Stadt « aus; die Sozialplanung wird dann oft als Ausgleich eingesetzt, um das soziale und kulturelle Zusammenlaben im Gemeinwesen noch aufrecht zu erhalten. Eine »Integrierte Sozialraumplanung " könnte beide Welten zusammenführen, wie ein Modellversuch in Erfurt zeigt.
\end{abstract}

Städte und Gemeinden sind damit beauftragt, gleichwertige Lebensbedingungen für alle Bevölkerungsgruppen zu schaffen. Es ist kaum notwendig zu belegen, dass diese im Raumordungsgesetz ( $§ 1$ Abs. 1) aber auch bereits im Grundgesetz (Art. 3) formulierte Zielstellung immer schwieriger zu verwirklichen ist. Die inzwischen wieder verstärkt sichtbare Abbildung von Armutsprozessen auf der Stadtlandschaft, die Abkopplung städtischer Teilräume, aber auch die Verödung ganzer Landstriche sprechen für sich: Es gibt sichtbare Tendenzen für eine weitere Polarisierung von Arm und Reich in städtischen Räumen (Farwick 2001; Kronauer 2002: 215 ff.; Häußermann 2004; Kronauer 2005).

Bei der Frage nach den Ursachen dominieren die bekannten Sachzwangrhetoriken: Die Klage über die sinkenden finanziellen Spielräume bei gleichzeitig steigender kommunaler Aufgabenbelastung mag häufig gerechtfertigt sein. Die problematischen Folgen vor allem die beschäftigungsbedingte Migration sind mit Blick auf bestimmte Regionen, wie sie vor allem im Osten der Republik zu finden sind, kaum zu bestreiten. In der öffentlichen Debatte werden die schwindenden Steuerungsund Handlungskapazitäten des lokalen Staates jedoch häufig allein »äußeren Umständen « angelastet. Die örtlichen (Fehl-) Entwicklungen erscheinen damit als direkte Folge dieser Umstände und nicht etwa als Resultat der Reaktion auf diese Umstände. Kommunale Haushaltsdebatten und Etatentscheidungen zeigen hingegen recht deutlich, dass durchaus Gestaltungsspielräume existieren, wenngleich diese sehr eingeschränkt sein können.

Welchen Prioritäten die Verwendung vorhandener Ressourcen folgt, wird aber immer noch vor Ort bestimmt. Das ist auch so gewollt; es entspricht dem Grundsatz der kommunalen Selbstverwaltung (Art. 28 Abs. 2 GG). Über die Entscheidungen von Stadt- oder Gemeinderäten hinaus spielen hierbei politische Präferenzen und Bewältigungslogiken ebenso eine Rolle wie gewachsene Organisationskulturen in der Kommunalverwaltung. Nicht zu vergessen ist der Einfluss bestimmter Interessenkoalitionen, deren konkrete Zusammensetzung von Ort zu Ort recht unterschiedlich ausfallen kann und die nicht immer leicht $\mathrm{zu}$ bestimmen sind. In der Summe entstehen ver-

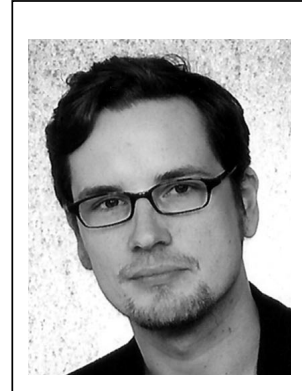

\section{Mario Rund ist Diplom-Sozial- pädagoge (FH); Praxis als Ge- meinwesenarbeiter; Lehraufträge (Fachhochschule Erfurt) zu Sozi- alraumorientierung, Sozialraum- planung, Sozialraumtheorie; derzeit Promotion (Universität Göttingen) zu Sozialraumstrate- gien und Gouvernementalität;} Fachberatung und Öffentlichkeitsarbeit bei LAG Soziale Stadtentwicklung und Gemeinwesenarbeit Thüringen.

E-Mail mario.rund@fh-erfurt.de

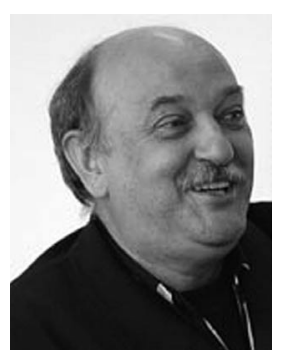

Prof. Dr. Ronald Lutz ist Hochschullehrer für Soziologie mit dem Schwerpunkt "Menschen in besonderen Lebenslagen « an der Fachhochschule Erfurt; Arbeitsgebiete: Armut und Reichtum, soziale Problemlagen, Armutsund Sozialberichterstattung, Ungleichheit u. a. E-Maillutz@fh-erfurt.de

schiedene stadtpolitische Profile, denen jeweils bestimmte Auffassungen oder »Theorien « darüber zugrunde liegen, wie eine Stadt oder eine Gemeinde angesichts wahrgenommener Herausforderungen und Gestaltungsoptionen zu entwickeln sei.

\section{"Unternehmen Stadt»}

Das in der Bundesrepublik vorherrschende Profil lässt sich als »unternehmerische Stadtpolitik « charakterisieren. Im Kontext der »Neuen Steuerung « folgt sie in ihren Rationalitäten und Organisationsweisen Unternehmen der freien Wirtschaft. Das »Unternehmen Stadt« definiert sich selbst im Wettbewerb zu anderen Städten. (u. a. Stratmann 1999:107 f.; Alisch 2002: 53 f.) Es ist in erster Line der Sicherung des Standorts, also der Herstellung und Pflege unternehmerischer Attraktivität verpflichtet (Ronneberger 2001; Ronneberger et al. 1999). Regionale Disparitäten und sozialräumliche Differenzierungsprozesse werden dadurch aber häufig eher gefördert als abgebaut. Denn den Bezugspunkt der unternehmerischen Stadt bilden vor allem potenzielle Investoren, Konsumfähige und vielleicht 
noch diejenigen, deren Ressourcen sich im Sinne von »sozialem Kapital« mobilisieren lassen (Mayer 2004: 73 f.).

Gegenüber derartigen Politiken, die sich theoretisch und legitimatorisch auf »regulierende Kräfte des Marktes « beziehen, haben sich in der 1990er Jahren eine Reihe von Politikansätzen entwickelt, die sich ihrer gemeinsamen Zielperspektive entsprechend unter »soziale Stadtentwicklung « zusammenfassen lassen. (Alisch 2002: 78) Ihnen liegt die Auffassung zugrunde, dass die Städte und Gemeinden die Folgen der gegenwärtigen Transformationsprozesse nur über eine aktive Politik bewältigen können, die eine Stadtentwicklung des sozialen Ausgleichs ermöglicht (ebd.).

Wirtschaftliche Orientierungen verlieren dabei keineswegs an Bedeutung; vielmehr gilt es diese mit sozialen und kulturellen Zielen - und im Übrigen auch mit ökologischen Standards - in Übereinstimmung zu bringen. Soziale Stadtentwicklung verspricht darüber hinaus eine höhere Sensibilität gegenüber Differenzentwicklung in städtischen Teilgebieten. Prekäre Lebenslagen werden dabei auch und vor allem in ihrer strukturellen Bedingtheit wahrgenommen.

Im Fokus steht daher nicht nur die baulich-technische Ausstattung oder die soziokulturelle Infrastruktur der Stadtteile, Quartiere und Sozialräume. Auch die Art des sozialen und interkulturellen Zusammenlebens, Bildungsund Qualifizierungsmöglichkeiten, Mitbestimmungs- und Teilhabechancen verschiedener Einwohnergruppen sowie lokale Bewältigungsressourcen gelten als wichtige Kriterien für den Entwicklungs- und Gestaltungsprozess.

Aus den aufgeführten Gründen ist die soziale Stadtentwicklung hinsichtlich der Zielperspektive, ausgleichende Lebensbedingungen herzustellen, nicht nur eine naheliegende, sondern notwendige stadtpolitische Orientierung. Eine in diesem Sinne zu verwirklichende soziale Stadtentwicklungspolitik stellt zweifellos hohe Anforderungen an die Akteure in Politik und Verwaltung und all jene, die beruflich oder auch ehrenamtlich »vor Ort « tätig sind. Die Kommunikation und Kooperation zwischen den Sektoren sowie Prozesse der Entscheidungsfindung und Entscheidungsumsetzung sind davon ebenso betroffen, wie die Erzeugung und der Austausch planungsrelevanten Wissens. Zudem wird neben dem Vorhandensein von konkreten Zuständigkeiten und Verantwortlichkeiten auch die grundsätzliche Bereitschaft vorausgesetzt, Ressortdenken und etablierte Bewältigungsroutinen kritisch zu prüfen und gegebenenfalls zu überwinden.

\section{Stadtplanung und Sozialplanung versöhnen}

Eine Antwort darauf, diesen Anforderungen auf struktureller Ebene gerecht zu werden, kann in der Integrierten Sozialraumplanung liegen. Bei diesem Ansatz, der gegenwärtig der Thüringer Landeshauptsstadt Erfurt als Modell vorliegt, werden Felder der Sozialplanung und Stadtplanung in einem Gesamtprozess sinnvoll vernetzt und re-strukturiert (vgl. Abbildungen Seite 16). Um eine bedarfsgerechtere Versorgung der Wohnbevölkerung und eine entsprechende Entwicklung von Sozialräumen zu erreichen, zielt dieses Modell nicht nur auf eine Optimierung des Zusammenspiels von Akteuren, Mitteln und Wissensbeständen. Das Modell wurde auch auf breiter fachlicher Basis entwickelt.

In einem über ein Jahr währenden Prozess der Recherche, des Austausches und der Diskussion konnten Fachperspektiven, aber auch Anregungen und Wünsche aus unterschiedlichen Funktionsbereichen zusammengeführt und verhandelt werden. Hierzu wurden mehrere Workshops angeboten, um sowohl die Politik, die Verwaltung und die Träger sozialer Dienste an dem Konzeptionsprozess einer möglichen Sozialraumplanung zu beteiligen. Zentrale Momente des Modells, die Vernetzung und Mo-

\section{ialraumplanung muss Politik, haft an einen Tisch bolen «}

deration der verschiedenen Interessen in den Planungsprozessen, konnte hier bereits anschaulich demonstriert werden.

Dabei wurden nicht nur disziplinäre und sektorale Grenzen überwunden. Das politisch-administrative System auf kommunaler Ebene, dessen Funktionsebenen durch funktionelle Hierarchien strukturiert sind, wurde kurzgeschlossen. So konnten die Vertreter der Politik, der Fachverwaltungen und der Erbringungsebene in direkten Kontakt treten. Damit war es möglich, Impulse aus Fachplanungen verschiedener Ressorts, der Sozialen Arbeit, der Stadtteil- und Gemeinwesenarbeit sowie aus sozialwissenschaftlichen Feldern in die Modellkonzeption einzubringen. Neue Verständigungs- und Austauschformen, welche das Modell erst etablieren soll, wurden auf diese Weise bereits in dessen Entstehungsprozess erprobt.

Vor dem Horizont dieser Erfahrungen erscheint es zum einen als durchaus realistisch, dass die Integrierte Sozialraumplanung sich gut implementieren lässt. Zum anderen unterstreichen diese Erfahrungen die Richtigkeit der Entscheidung, die Modellentwicklung externen Sachverstand anzuvertrauen und als Moderationsprozess anzulegen. Bei einer Aufgabe, von derart grundsätzlichem Charakter, wie sie die Verräumlichung und das ReArrangement der kommunalen Sozialplanung darstellt, liegen die Vorteile dieses Vorgehens auf der Hand: Die bei Binnenbemühungen nachvollziehbare Zurückhaltung lohnabhängig Beschäftigter, Probleme in ihren Arbeitszusammenhängen zu benennen oder aber auch Kritik implizierende Verbesserungsvorschläge zu formulieren, musste kaum befürchtet werden. Und statt lediglich eine in Auftrag gegebene Expertise zu erstellen und abzuliefern, wurde der Modellentwurf von Anbeginn an unter der aktiven Mitarbeit der relevanten Akteure erarbeitet. Das Modell einer Integrierten Sozialraumplanung zeigt daher auch die Offenheit und Bereitschaft in Politik und Verwaltung, an der Verbesserung einer oft widersprüchlichen Praxis teilzuhaben, in der die eigenen Vorstellungen nicht selten hinter den Möglichkeiten zurückbleiben. 

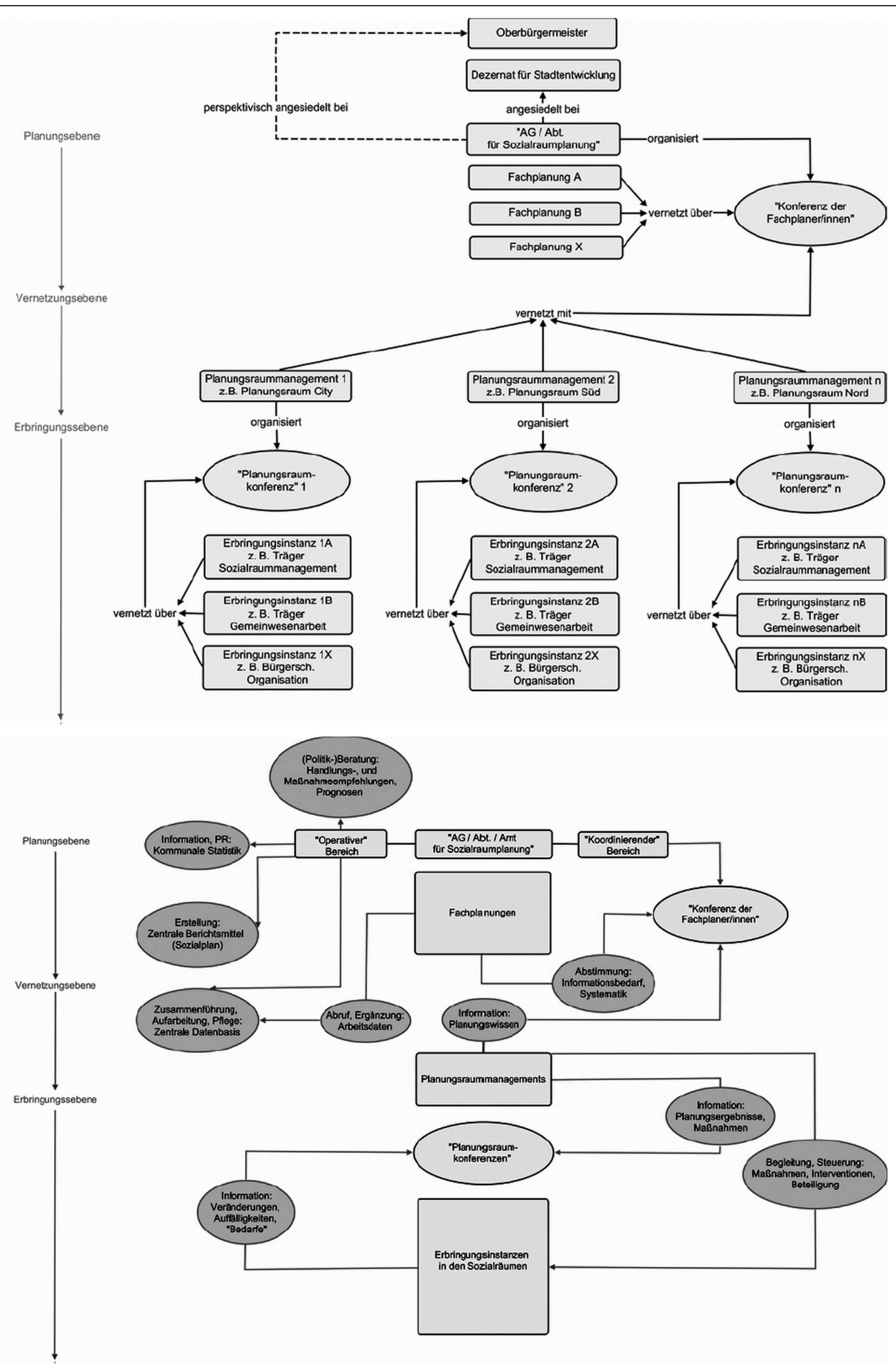

Die Integrierte Sozialraumplanung, wie sie in der Thüringer Landeshauptstadt Erfurt erprobt wird, braucht temporäre und kontinuierliche Austauschforen für Politik, Verwaltung, Leistungserbringer und Bürgerschaft. Die obere Grafik zeigt die dabei zu berücksichtigenden kommunikativ-strukturellen Aspekte, die untere Abbildung verdeutlicht die Aufgabenverteilung.

Quelle: Ronald Lutz (Fachhochschule Erfurt): Integrierte Sozialraumplanung in der Thüringer Landeshauptstadt Erfurt. Bericht vom 26. Oktober 2007. Seite $21 \mathrm{f}$. 
Das Modell der Integrierten Sozialraumplanung wurde auf Basis spezifischer Bedingungen und Ressourcen einer konkreten Stadt konzipiert. Bei der Frage nach der Verallgemeinerungsfähigkeit ist dies zu berücksichtigen. Zudem lässt sich ein solches Modell nur mit dem nötigen politischen Willen und keineswegs gegen den Widerstand der Praxis einführen. Ein von der Politik artikuliertes Problembewusstsein und ein auf breiter Ebene konstatierter Veränderungsbedarf sind daher nicht nur Vorbedingungen für die Entwicklung des Modells; sie bilden wichtige Anknüpfungspunkte für den immanenten Moderationsund Mitgestaltungsprozess. Zudem müssen die erforderlichen Mittel für die Implementierungsphase in der Haushaltplanung angemessen berücksichtigen werden.

Die Integrierten Sozialraumplanung geht, um eine zielgerichtete Stadtentwicklungspolitik umzusetzen, von einem weiten Begriff des Sozialen aus, der sich an den Konzepten der »Lebenslage« festmachen lässt. Das sozialwissenschaftliche Lebenslagenmodell (u. a. Weisser 1978; Nahnsen 1992; Engels 2008), das sich in der Sozialberichterstattung bereits als brauchbar erwiesen hat, ist ein geeigneter Ausgangspunkt planerischer Prozesse. Dimensionen der Lebenslage von Menschen sind $\mathrm{u}$. a.: Wohnen, Bildung, Arbeit, Mobilität, Gesundheit, Ernährung, soziale Beziehungen, Partizipation, Kultur und Freizeit und subjektives Wohlbefinden.

Das Projekt Integrierte Sozialraumplanung zielt grundsätzlich darauf, in der Kooperation mit Politik und Verwaltung, sozialen Trägern sowie den Einwohnerinnen und Einwohnern (bzw. deren legitimierten Vertretungen) ein tragfähiges Planungsmodell zu entwickeln, das die sich im Zeitverlauf verändernde Situation und Bedarfsstruktur unterschiedlicher Bevölkerungsgruppen kleinräumiger, integrierter und damit lebenslagennäher abzubilden vermag. Dabei setzt es auf Mitbestimmung und auf Teilhabe aller Akteure in verschiedenen Stufen der Planungsprozesse.

Das Modell greift dabei die klassischen Elemente einer Bedarfsanmeldung von unten auf, Instrumente, in denen sich kommunale Akteure sowie Einwohnerinnen und Einwohner zusammensetzen, um gemeinsame Vorstellungen für die Entwicklung der Sozialräume erarbeiten. Genannt werden dabei explizit »Sozialraumkonferenzen« auf der Ebene der Sozialräume selbst sowie »Planungsraumkonferenzen«, die mehrere soziale Räume umfassen können. Die städtischen Planungsroutinen sollen dadurch besser auf die örtlichen Besonderheiten abgestimmt werden. Gleichzeitig wird eine neue Instanz geschaffen, welche die Inkompatibilität zwischen durchaus dynamischen Sozialraumgrenzen und den eher starren administrativ definierten Planungsraumzuschnitten überbrücken kann: Sogenannte Planungsraummanager und Planungsraummanagerinnen vertreten die in den jeweiligen Planungsräumen zusammengefassten Sozialräume auf den Planungsraumkonferenzen. Sie transportieren und vermitteln auf diese Weise planungsrelevantes Wissen aus der Bevölkerung und von den sozialen Trägern in die Fachabteilungen. Damit werden die unterschiedlichen Lebensbedingungen und Lebenszusammenhänge, die sich auf vielfältigste Lebenslagebereiche der Menschen auswirken, in einem fachübergreifenden und kooperativen Planungsprozess gewürdigt, reflektiert und zur Grundlage der bedarfsgerechten und ausgleichenden Gestaltung von städtischen Teilräumen genommen.
Planung wird somit zu einem Prozess, der auf der Basis von permanenten Aushandlungsprozessen zwischen den Menschen in den Sozialräumen, den sozialen und ökonomischen Akteuren und einer gestaltungsbereiten Politik ermöglicht wird. Integrierte Sozialraumplanung ist dann eine Stadtentwicklungsplanung mit allen Akteuren; sie ist auf diese Weise Teil und Vorraussetzung einer sozialen Stadtentwicklung, die beteiligen, ermöglichen und entwickeln will.

\section{Literatur}

Alisch, Monika 2002: Soziale Stadtentwicklung. Widersprüche, Kausalitäten und Lösungen. Opladen.

Engels, Dietrich 2008: Lebenslagen. In: Maelicke, Bernd (Hg.). Lexikon der Sozialwirtschaft. Baden-Baden, S. 643-646.

Farwick, Andreas 2001: Segregierte Armut in der Stadt. Ursachen und soziale Folgen der räumlichen Konzentration von Sozialhilfeempfängern. Opladen.

Häußermann, Hartmut 2004: »Problembehaftete« Gebiete. In: Odierna, Simone \& Berendt, Ulrike (Hg.). Gemeinwesenarbeit. Entwicklungslinien und Handlungsfelder. Neu-Ulm, S. 129-139.

Kronauer, Martin 2002: Exklusion. Die Gefährdung des Sozialen im hoch entwickelten Kapitalismus. Frankfurt am Main, New York.

Kronauer, Martin 2005: Ausgrenzung und physischsozialer Raum. In: Anhorn, Roland \& Bettinger, Frank (Hg.). Sozialer Ausschluss und Soziale Arbeit. Positionsbestimmungen einer kritischen Theorie und Praxis Sozialer Arbeit. Wiesbaden, S. 167-184.

Mayer, Margit 2004: Vom Versprechen lokaler Kohäsion. Blindstellen in der intentionalen Debatte. In: Kessl, Fabiano \& Otto, Hans-Uwe (Hg.). Soziale Arbeit und Soziales Kapital. Zur Kritik lokaler Gemeinschaftlichkeit. Wiesbaden, S. 63-78.

Nahnsen, Ingeborg 1992: Lebenslagenvergleich. Ein Beitrag zur Vereinigungsproblematik. In: Henkel, Heinrich \& Merle, Ulrich (Hg.):. Magdeburger Erklärung. Neue Aufgaben in der Wohnungswirtschaft. Beiträge zum deutschen Einigungsprozess. Kölner Schriften zur Sozial- und Wirtschaftspolitik, Bd. 23. Köln, S. 101-144.

Ronneberger, Klaus 2001: Konsumfestungen und Raumpatrouillen. In: Becker, Jochen (Hg.). Bignes? Size does matter. Image/Politik. Städtisches Handeln Kritik der unternehmerischen Stadt. Berlin, S. 28-41.

Ronneberger, Klaus; Lanz, Stephan \& Jahn, Walter 1999: Die Stadt als Beute. Bonn.

Stratmann, Bernhard 1999: Stadtentwicklung in globalen Zeiten. Lokale Strategien, städtische Lebensqualität und Globalisierung. Basel.

Weisser, Gerhard 1978: Beiträge zur Gesellschaftspolitik. ausgewählt und hg. von Siegfried Katterle, Mudra, Wolfgang und Neumann, Lothar F. Göttingen. 